\title{
Comparison of Two Software Tools for Damage Identification: Gradient-based vs. Case-based Approach
}

\author{
Przemysław Kołakowski ${ }^{1}$, Luis E. Mujica ${ }^{2}$, Josep Vehí ${ }^{2}$ \\ ${ }^{1}$ Institute of Fundamental Technological Research, Swietokrzyska 21, 00-049 Warsaw, Poland, \\ pkolak@ippt.gov.pl \\ ${ }^{2}$ University of Girona, Edifici P-IV, E-17071 Girona, Spain, lemujica@eia.udg.es, vehi@eia.udg.es
}

Keywords: damage identification, inverse dynamic problem, software tools

\begin{abstract}
Two alternative software tools for damage identification are presented. The first tool, developed on the basis of the Virtual Distortion Method (VDM), takes advantage of an analytical formulation of the damage identification problem. Consequently, gradient-based optimization method is applied to solve the resulting dynamic inverse problem in time domain. Finite element model of the structure is necessary for the VDM approach. The second tool utilizes the Case-Based Reasoning (CBR) for damage identification. This method consists in i) extracting principal features of the response signal by wavelet transform, ii) creating a base of representative damage cases, iii) organizing and training the base by neural networks, and finally iv) retrieving and adapting a new case (possible damage) by similarity criteria. Basic description of both approaches is given. A comparison of numerical effectiveness, in terms of accuracy and computational time, is provided for a simple beam structure. Advantages and weaknesses of each approach are highlighted.
\end{abstract}

\section{Introduction}

The methods described in this paper belong to the low-frequency-based methods of damage identification. The incentive for developing them was the 5FP project PiezoDiagnostics (PD), dedicated to identification of corrosion in pipelines. In this project, damage identification tools were based on the phenomenon of elastic wave propagation in structures. The waves of low (below 1 $\mathrm{kHz}$ ) frequency were generated and captured by piezoelectric transducers. Structural responses (signatures) to the piezo-generated excitation were analyzed and anomalies in the responses were observed due to various types of damage.

Basically, two independent approaches, leading eventually to two software packages have been developed in the PD project. The first approach - Virtual Distortion Method (VDM), developed by the IFTR, uses gradient-based optimization techniques and the other approach - Case-Based Reasoning (CBR), developed by the University of Girona (UdG), uses the wavelet transform for signal processing and neural networks to match cases by similarity criteria.

Overviews of the VDM and CBR are given, respectively. Identification algorithms for each approach and comments on critical factors, highly influencing the success or failure of damage identification, are presented. A numerical example of a simple beam structure, handled by both methods, is demonstrated. Final comments summarize the comparison between the two approaches.

\section{Characteristics of the Two Approaches}

VDM. The Virtual Distortion Method has been developed for many years by the IFTR. It may be simply classified as a fast reanalysis technique (cf. [1]). This method is very efficient if we know an original response of the structure and then want to introduce some modifications to its behaviour 
without repeating the whole analysis. With the VDM we are able to solve various problems of structural mechanics e.g. progressive collapse, structural remodelling, damage identification, damping of vibration, adaptive structure design and other.

As mentioned, VDM is used for modelling of local structural modifications. This is achieved by introducing a corresponding virtual distortion $\varepsilon^{\mathrm{o}}$, which is an initial strain, imposed locally in an element of a discrete (truss) or discretized (beam) structure. The VDM reanalysis can be performed very fast (with no iterations due to introducing an initial strain) because of the so-called influence matrix D, which is always created as a basis for all computations. The influence matrix defines all local-global inter-relations for a structure, including boundary conditions. It is obtained by successive imposition of unit virtual distortion in every element of the structure. Green's functions are the analogy to the influence matrix for continuum. Detailed description of the VDM and its applications can be found in [2].

Let us briefly describe (cf. [3]) the inverse dynamic problem of damage identification. The objective is to determine damage location and intensity, both specified by the damage vector $\mu_{i}$, whose components correspond to the designed discretization of the structure (finite elements). The vector $\mu_{i}$, being the stiffness degradation ratio (the quotient of the current $\mathrm{E}_{\mathrm{i}}$ and initial $\mathrm{E}_{\mathrm{i}}$ Young's modulus), is expressed in terms of strains $\varepsilon_{\mathrm{i}}(\mathrm{t})$ and virtual distortions $\varepsilon_{\mathrm{i}}^{0}(\mathrm{t})$, as follows:

$$
\mu_{i}=\frac{E_{i}}{E_{i}^{\prime}}=\frac{\varepsilon_{i}(t)-\varepsilon_{i}^{0}(t)}{\varepsilon_{i}(t)}
$$

Please note that the damage vector $\mu_{i}$ is time-independent in contrast to strains and virtual distortions.

Let us define the objective function as the mean-square distance $f_{A}$ between the measured response $\varepsilon_{\mathrm{A}}^{\mathrm{M}}(\mathrm{t})$ and numerical response $\varepsilon_{\mathrm{A}}(\mathrm{t})$, collected from the sensor locations $\mathrm{A}$. The responses are due to the known excitation $\varepsilon_{\alpha}^{0}(\tau)$, generated in the actuator location $\alpha$. The numerical response is a superposition of the component $\varepsilon_{\mathrm{A}}^{\mathrm{L}}(\mathrm{t})$ expressing an intact response and the component $\varepsilon_{\mathrm{A}}^{\mathrm{R}}(\mathrm{t})$ adapting the intact response to the measured response $\varepsilon_{\mathrm{A}}^{\mathrm{M}}(\mathrm{t})$ by proper setting of virtual distortions $\varepsilon_{\mathrm{j}}^{0}(\mathrm{t})$ in possible damage locations $\mathrm{j}$ (potentially the whole structure). The influence matrix D, built at start of numerical simulation, plays the crucial role in VDM-based modelling. Thus the objective function is expressed as:

$$
\mathrm{f}=\sum_{\mathrm{A}} \mathrm{f}_{\mathrm{A}}=\sum_{\mathrm{A}} \sum_{\mathrm{t}}\left[\mathrm{d}_{\mathrm{A}}(\mathrm{t})\right]^{2}
$$

where

$$
\begin{aligned}
\mathrm{d}_{\mathrm{A}}(\mathrm{t}) & =\varepsilon_{\mathrm{A}}^{\mathrm{M}}(\mathrm{t})-\varepsilon_{\mathrm{A}}(\mathrm{t})=\varepsilon_{\mathrm{A}}^{\mathrm{M}}(\mathrm{t})-\left[\varepsilon_{\mathrm{A}}^{\mathrm{L}}(\mathrm{t})+\varepsilon_{\mathrm{A}}^{\mathrm{R}}(\mathrm{t})\right]=\varepsilon_{\mathrm{A}}^{\mathrm{M}}(\mathrm{t})-\sum_{\mathrm{t}}\left[\varepsilon_{\mathrm{A}}^{\mathrm{L}}(\mathrm{t})+\varepsilon_{\mathrm{A}}^{\mathrm{R}}(\mathrm{t})\right]= \\
& =\varepsilon_{\mathrm{A}}^{\mathrm{M}}(\mathrm{t})-\sum_{\tau \leq t} \sum_{\tau^{\prime} \leq \tau}\left[\sum_{\alpha} \mathrm{D}_{\mathrm{A} \alpha}\left(\tau-\tau^{\prime}\right) \varepsilon_{\alpha}^{0}\left(\tau^{\prime}\right)+\sum_{\mathrm{j}} \mathrm{D}_{\mathrm{Aj}}\left(\tau-\tau^{\prime}\right) \varepsilon_{\mathrm{j}}^{0}\left(\tau^{\prime}\right)\right] .
\end{aligned}
$$

The inverse dynamic problem of damage identification consists in finding the minimum of the function $\mathrm{f}$, with $\mu_{\mathrm{i}}$ as design variables. In view of Eq. 1, the following constraints on $\mu_{\mathrm{i}}$ have to be considered: 
In order to solve the optimization problem, a gradient-based approach is applied, with the following analytical gradient calculated from the Eqs. 2a, 2b:

$$
\nabla \mathrm{f}_{\mathrm{k}}=\frac{\partial \mathrm{f}}{\partial \mu_{\mathrm{k}}}=\sum_{\mathrm{A}} \frac{\partial \mathrm{f}_{\mathrm{A}}}{\partial \mu_{\mathrm{k}}}=-2 \sum_{\mathrm{A}} \sum_{\mathrm{t}} \mathrm{d}_{\mathrm{A}}(\mathrm{t})\left[\sum_{\tau \leq t} \sum_{\tau} \sum_{\mathrm{j}} \mathrm{D}_{\mathrm{Aj}}\left(\tau-\tau^{\prime}\right) \frac{\partial \varepsilon_{\mathrm{j}}^{0}\left(\tau^{\prime}\right)}{\partial \mu_{\mathrm{k}}}\right] \text {, }
$$

Having determined the above gradient of the objective function, the following optimization step is proposed:

$$
\mu_{\mathrm{i}}=\mu_{\mathrm{i}}-\frac{\gamma \mathrm{f}\left(\mu_{\mathrm{i}}\right)}{\nabla \mathrm{f}_{\mathrm{i}}^{\mathrm{T}} \nabla \mathrm{f}_{\mathrm{i}}} \nabla \mathrm{f}_{\mathrm{i}}
$$

where $\gamma=$ const. and $f\left(\mu_{i}\right)$ is the current value of the objective function. Then, the calculation of the objective function and its gradients for the modified structure response can be performed in the next iteration until termination criterion is met.

CBR. This section describes the UdG-developed methodology (cf. [4]) of damage identification (location, size and intensity), using the knowledge-based reasoning and soft-computing techniques. First, a model is used to simulate responses of damaged structures and to generate case libraries. Using the Self Organizing Maps (SOMs, cf. [5]) as classification tool, an initial casebase is built. This casebase is used in diagnosing future situations by analogy (see Fig. 1). In order to reduce the number of input signals to the SOM (it is required to have appropriate classification accuracy), the Wavelet Transform (WT, cf. [6]) has been utilized to extract features from the measured signal, retaining most of the intrinsic information. When the system is in operation mode, each new experience is retained once the damage has been detected.

Case Libraries. Building the case library is done from a model or several models of the structure, simulating the dynamic response to a given excitation in the presence of one or several defects. Each library of cases is a data structure, which contains information about the simulated defect in the structure (location, size and intensity) and the simulated dynamic response.

Casebase Building. The casebase is an array in memory of all cases, which facilitates the search of the case, being the most similar to the current problem. In the proposed methodology, the casebase is a SOM. It retrieves the group of stored cases with similar characteristics. Each case to save in the casebase is defined by the defect of the structure and the minimal representation of the damaged structure response. These principal features are extracted from coefficients of the WT applied to the dynamic response. The wavelet coefficients are computed from each selected case. The coefficients at the same position in different cases are considered as samples of independent random variables. Therefore, having in mind the Central Limit Theorem, each variable is approximately normally distributed. The peak probability values and the maximal wavelet coefficients occur at the same positions, which determine the midpoints of clusters. This pattern of clusters contains relevant signal information. Later on, each feature is determined as the square root of the energy of the wavelet coefficients in the corresponding cluster. This set of cases (defect of the structure and principal features of the signal) is used to build a casebase. Fig. 2 shows the process of feature extraction and building the casebase. After having the set of cases generated (defect and the principal features of the dynamic response), the organization of the data in memory for its recovery at the indicated moment is executed. A SOM is created and trained. This SOM has $l$ neurons (one for each feature) in the 1D input layer and $m^{*} n$ clusters or neurons in the 2D output layer. In each cluster, the network organizes the cases with similar characteristics. 


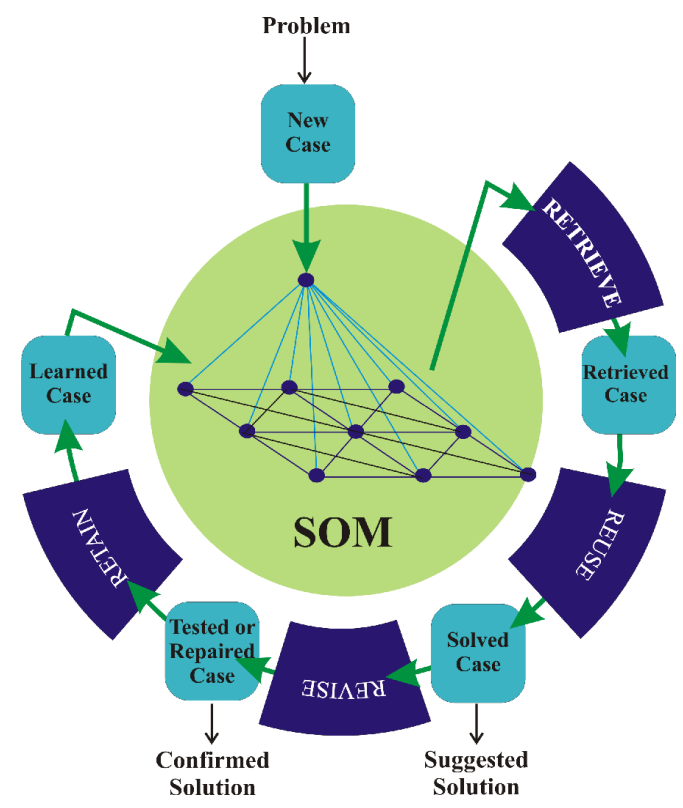

Fig. 1 Proposed CBR cycle

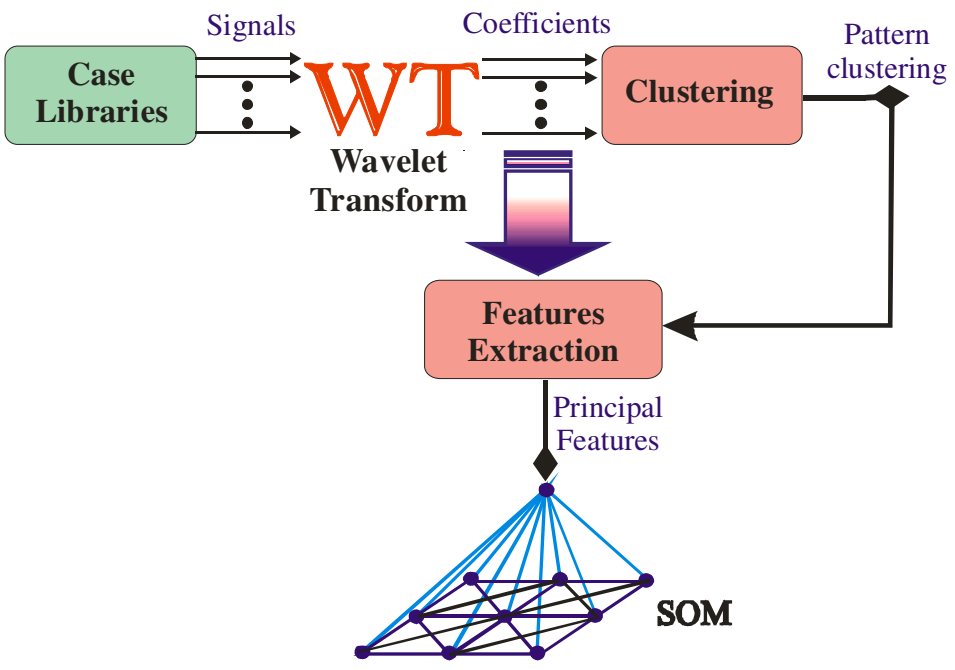

Fig. 2 Casebase building

Retrieving. Checking the methodology or putting the system in operation mode, can be done by numerical simulations, lab-testing and field-testing with real structures in their normal working conditions. Dynamic response of the structure is collected. From this signal, the principal features are extracted using the clustering pattern previously defined. From these features the SOM retrieves a set of stored cases with similar characteristics.

Adapting. Using the previously retrieved cases, the first approach to adapt the solution and locate the defect is based on the concept of statistical mode, which is the defect that occurs most frequently in a set of retrieved cases. In order to calculate the size and the intensity of the defects, the weighted average is computed, using the data histogram (or distances) of the retrieved step as weighting coefficients. 


\section{Specific Features of the Two Approaches}

VDM algorithm. The VDM-based identification algorithm performs the following steps:

1. Complete modal analysis,

2. Excite the beam with the windowed sine burst (isolated eigenmode providing smooth vibration response),

3. Choose a low (below $1 \mathrm{kHz}$ ) eigenfrequency resulting in the highest value of the objective function,

4. Look at initial gradients (rough indication of potential damage locations),

5. Perform optimization

a) modify damage coefficient according to Eq. 5,

b) take heuristic care of the constraints, expressed by Eq. 3 .

VDM settings. The cost of computation of the gradients, expressed by Eq. 4, is high. It is influenced by the initial parameters assumed in analysis, especially by the number of time steps in Newmark algorithm and extension of the potentially damaged zone of the structure (the number of finite elements for which gradients are calculated). The higher number of time steps leads to some point to more accurate results, but at a certain stage the saturation point can be determined, beyond which no significant improvement is observed. This is the right compromise between the accuracy and computational speed. The higher number of finite elements considered as potentially damaged slows down the computations considerably. Therefore any hint based on engineering judgement on where the damage may be located is valuable as it narrows the zone. One should remember however that if we expect a small damage, the FE size should roughly correspond to our initial guess. Otherwise the small damage will not be detected.

CBR algorithm. The CBR-based identification algorithm performs the following steps:

1. Choose a structure to study,

2. Define what kind of damage should be identified (size and intensity),

3. Choose a set of case libraries, which contain previous simulations of various patterns of structural damage,

4. Build a casebase from the selected libraries,

5. Upload the waveform detected by the sensor in a supposedly damaged structure,

6. Retrieve the most similar cases from the casebase,

7. Adapt the previous solutions to propose a new solution,

8. Generate the outcome report and display the identified damage of the structure,

9. Retain the new solution as a part of a new case once it has been confirmed or validated.

CBR settings. In order to get a meaningful identification result by $\mathrm{CBR}$, one should build representative casebases. This means that a casebase should correspond to the expected type of damage as far as the number of damage locations, their extension and intensity are concerned. Properly built casebase, reflecting the features of a real damage, will have impact on the quality of the identification results. Another factor influencing the quality, but also computational time is the number of cases stored in a casebase. It seems that the higher number of cases implies better results, but a saturation point should be observed here as well, similarly to time steps in the VDM approach. Credibility of a casebase should be assured by calibration of the numerical model used for generation of cases of damaged structure responses. 


\section{Numerical Example}

For demonstration of effectiveness of the PD software a cantilever beam structure, depicted in Fig. 3 , has been chosen. The beam is $98 \mathrm{~cm}$ long, $2 \mathrm{~cm}$ wide, $0.5 \mathrm{~cm}$ high, made of aluminium, discretized into 49 finite elements. The actuator is mounted in the middle of the beam in order to avoid the problem of false damage identification (see Section 4). It is excited with a 2.5-cycle, windowed sine burst of the frequency $491 \mathrm{~Hz}$, corresponding to the 7th eigenmode of the beam.

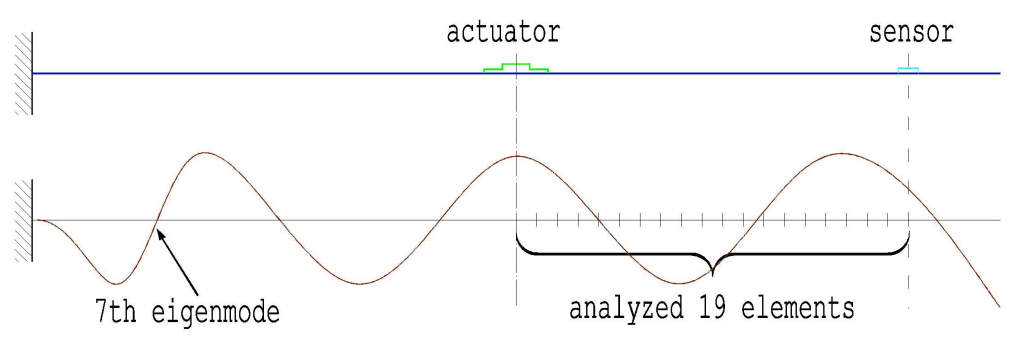

Fig. 3 Cantilever beam analyzed by VDM and CBR

The whole zone between the actuator and the sensor, comprising 19 elements, is inspected. The damage in the structure has been simulated numerically by reduction of Young's modulus in selected finite elements. Three damage cases, summarized in Table 1, have been considered.

Table 1 Assumed damage cases

\begin{tabular}{|l|c|c|c|}
\hline \multirow{2}{*}{$\begin{array}{l}\text { Element chosen as } \\
\text { damaged }\end{array}$} & \multicolumn{3}{|c|}{ Damage intensity coefficient $(1-\mu)$} \\
\cline { 2 - 4 } Element No. 5 & Damage case 1 & Damage case 2 & Damage case 3 \\
\hline Element No. 10 & 0.0 & 0.3 & 0.3 \\
\hline Element No. 15 & 0.0 & 0.0 & 0.2 \\
\hline
\end{tabular}

Table 2 presents computational times needed for obtaining a solution by the VDM-based identification procedure for each damage case. In the gradient-based optimization process, a drop of the objective function by orders of magnitude is monitored. It is clear that the more accurate solution is required, the more time is needed to obtain it. The VDM-based computations have been accomplished by a PC equipped with a $3.0 \mathrm{GHz}$ processor.

Table 2 Computational times for VDM

\begin{tabular}{|l|c|c|c|}
\hline \multirow{2}{*}{$\begin{array}{l}\text { VDM-formulated } \\
\text { objective function }\end{array}$} & \multicolumn{3}{|c|}{ Computational time [min] } \\
\cline { 2 - 4 } & Damage case 1 & Damage case 2 & Damage case 3 \\
\hline Drop by 1 order & 12 & 15 & 16 \\
\hline Drop by 2 orders & 89 & 72 & 69 \\
\hline Drop by 3 orders & 327 & 444 & 283 \\
\hline Drop by 4 orders & 947 & 1019 & 760 \\
\hline
\end{tabular}

Table 3 presents analogous computational times needed by the CBR-based identification procedure. The constructed casebases are as follows:

- Casebase I (one defect): 127 structural responses of simulated defects on every element between the actuator and sensor with the thickness reduction of $10 \%, 20 \%, 30 \%, 40 \%, 50 \%$ and $60 \%$. 
- Casebase II (two defects): 630 structural responses of simulated defects on two separated (neighboring as a special case) elements between the actuator and sensor with the thickness reduction of $10 \%, 30 \%$ and $50 \%$.

- Casebase III (three defects): 1336 structural responses of simulated defects on three separated elements between the actuator and sensor with the thickness reduction of $10 \%, 30 \%$ and $50 \%$.

The CBR-based computations have been accomplished by a PC equipped with a $2.4 \mathrm{GHz}$ processor.

Table 3 Computational times for CBR

\begin{tabular}{|l|c|c|c|}
\hline \multirow{2}{*}{ CBR casebases } & \multicolumn{3}{|c|}{ Computational time [min] for all damage cases } \\
\cline { 2 - 4 } & Library building & Training & Total \\
\hline Casebase I & 1.4 & 0.1 & 1.5 \\
\hline Casebase II & 6.5 & 1.9 & 8.4 \\
\hline Casebase III & 12.6 & 4.8 & 17.4 \\
\hline Casebase I+II & 7.9 & 1.7 & 9.6 \\
\hline Casebase I+II+III & 20.6 & 19.9 & 40.5 \\
\hline
\end{tabular}

Fig. 4 presents VDM (left) vs. CBR (right) results for the three assumed damage cases. The vertical axis shows the damage intensity coefficient $(1-\mu)$ for VDM plots and the probability of damage location for CBR plots, therefore the graphics cannot be compared directly.
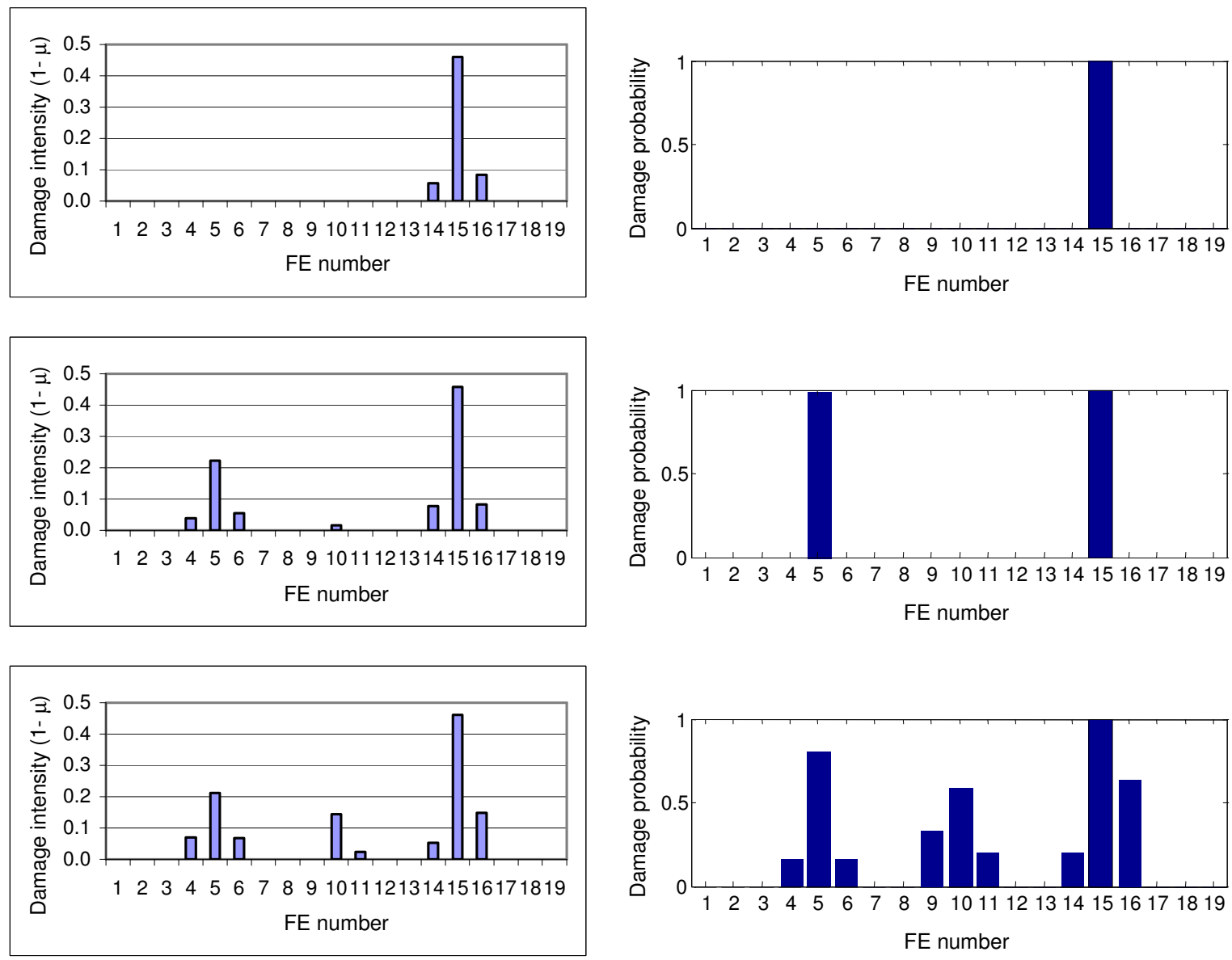

Fig. 4 VDM (left) vs. CBR (right) results for damage cases 1 (top), 2 (middle), 3 (bottom) 


\section{Conclusions}

The first approach, developed by the IFTR, uses the Virtual Distortion Method for modelling damage and standard optimization techniques for identifying it. The defined objective function refers to a mean square strain measure, which is then minimized in the optimization process. So, the VDM-based approach looks at strains, which may be physically measured e.g. by piezo-patches mounted on a beam. The identification results are always presented in deterministic manner. The advantage of the VDM approach is a relatively high accuracy of identification (having in mind the complexity of the inverse problem). The drawback of the method is high numerical cost involved due to costly calculation of the objective function gradients.

The alternative approach has been developed by the UdG and uses Case-Based Reasoning for damage identification. The proposed software tool may be described as efficient data processing machine. The CBR-based approach requires numerous input in terms of structural responses in order to create a library of various damage cases. Then is utilizes soft-computing methods (neural networks) for training the library of cases (building self-organizing maps). A new case is identified on the basis of previously stored and trained data. The identification results are always presented in probabilistic manner. The advantage of the CBR approach is that once the library of cases is built, the identification of a new case is almost immediate, whereas with the VDM approach the identification for a new case implies starting the procedure from scratch. The disadvantage of the CBR approach is that the quality of solution is highly influenced by the choice of a proper casebase. If the real number of damage locations is known (rarely the case) and matched with the proper casebase, the identification result will be accurate (see Fig. 4). Otherwise (e.g. using the combined casebase I+II+III, not focused on a certain defect type) only a rough defect indication will be given.

\section{Acknowledgements}

This work was financially supported by the 5FP EU Growth Project No. G1RD-2001-00659 PIEZODIAGNOSTICS - Smart Structural Diagnostics using Piezo-generated Elastic Waves; partners: CEGELEC, TWI, ATKINS, CEDRAT, CDRiA, CIMNE, IFTR, ECL, ALSTOM.

\section{References}

[1] Akgun M. A., Garcelon J. H., Haftka R. T. (2001) Fast Exact Linear and Non-linear Structural Reanalysis and the Sherman-Morrison-Woodbury Formulas, International Journal for Numerical Methods in Engineering, 50, pp. 1587-1606

[2] Holnicki-Szulc J., Gierlinski J. T. (1995) Structural Analysis, Design and Control by the Virtual Distortion Method, J. Wiley \& Sons, Chichester, U.K.

[3] Kolakowski P., Zielinski T.G., Holnicki-Szulc J. (2004) Damage Identification by the Dynamic Virtual Distortion Method, Journal of Intelligent Material Systems and Structures, vol. 15(6), pp. 479-493

[4] Mujica L.E., Vehi J., Rodellar J., Garcia O., Kolakowski P. (2004) Hybrid Knowledge Based Reasoning Approach for Structural Assessment, Proc. of the 2nd European Workshop on Structural Health Monitoring, 7-9 July, Munich, Germany

[5] Bose N.K., Liang P. (1996) Neural Networks Fundamentals with Graphs, Algorithms, and Applications, McGraw-Hill, Inc., U.S.

[6] Mallat S. (1999) A Wavelet Tour of Signal Processing, 2nd ed., Academic Press 